\title{
Efficient functionalization of poly(styrene) beads immobilized metal nanoparticle catalysts for the reduction of crystal violet
}

\author{
EAGAMBARAM MURUGAN* and PARAMASIVAM SHANMUGAM \\ Department of Physical Chemistry, School of Chemical Sciences, University of Madras, Maraimalai Campus, Guindy, \\ Chennai 600025, Tamil Nadu, India
}

MS received 26 August 2014; revised 24 September 2014

\begin{abstract}
Three types of new bead-shaped heterogeneous nanoparticle (NP) catalysts were synthesized by simplified procedures and studied for continuous reduction of crystal violet (CV) dye. The stabilizing agent, viz., 2-acryloxyethyltrimethyl ammonium chloride (PAC) was functionalized efficiently onto the surface of insoluble poly(styrene)-co-(vinyl benzyl chloride) beads (PS-PVBC) through surface-initiated atom transfer radical polymerization (SI-ATRP) to obtain insoluble bead matrix having intense surface functional groups labelled as PSPVBC- $g$-PAC. These PS-PVBC-PAC beads in turn were used as a common matrix for individual immobilization of AgNPs, AuNPs and PdNPs by following the simple chemical reaction/reduction methods to yield the corresponding bead-shaped heterogeneous NP catalysts, viz., PS-PVBC-g-PAC-AgNPs, PS-PVBC-g-PAC-AuNPs and PS-PVBCg-PAC-PdNPs. These catalysts were characterized by UV-Vis, FT-IR, SEM/EDAX and HRTEM techniques. The catalytic activity of these three types of catalysts were examined through the reduction of $\mathrm{CV}$ using $\mathrm{NaBH}_{4}$ as a reducing agent and it was observed that all these catalysts effectively accelerated the reaction. The superior catalyst, viz., PS-PVBC-g-PAC-AuNPs was again used for detail kinetic studies of the same reduction reaction.
\end{abstract}

Keywords. Heterogeneous nanocatalyst; atom radical polymerization reaction; reduction; crystal violet; polymer beads.

\section{Introduction}

It is well known that the metal nanoparticles (NPs) are of great interest owing to its potential applications in the sizedependent biomedical, electronic, optical, magnetic and catalytic materials. ${ }^{1}$ The field of nanocatalysis has undergone an explosive growth during the past decade, both in homogeneous and heterogeneous catalyses. The first report of polymer stabilized metal NPs appeared in $1998^{2}$ and suggesting an effective homogeneous catalyst. However, these homogeneous catalysts were not obtained with high yield during synthesis in aqueous medium. The recovery of such type of homogeneous catalysts has normally been carried out by dialysis, magnetic separation, precipitation and ultracentrifugation methods. ${ }^{3}$ However, again these methods are practically tedious and possess several limitations. More importantly, applications of homogeneous NP catalyst in organic reactions are not considered economical owing to their constraint in reuse. With a view to avoid all the demerits experienced in the homogeneous NPs catalyst, researchers have introduced an alternative catalyst, viz., 'heterogeneous nanoparticle catalyst'. 4 This catalyst was generally prepared by immobilization of metal NPs onto the insoluble polymer-supported matrices. Therefore, the research in stabilization/immobilization of metal NPs onto the surface of

\footnotetext{
*Author for correspondence (dr.e.murugan@gmail.com)
}

polymer bead matrix was growing very fast from earlier to till date.

Today, NP immobilized/stabilized polymer-supported heterogeneous catalyst has found a variety of applications due to the combination of physical and chemical properties of the NPs with the tunable solubility and surface reactivity of the polymer templates. The synthesis of heterogeneous catalyst involves immobilization of transition metal NPs on various solid matrices. Many solid support inorganic materials such as silica, ${ }^{5}$ alumina, ${ }^{6}$ metal oxides ${ }^{7}$ and few organic support materials such as carbon, ${ }^{8}$ poly(styrene) matrix ${ }^{9,10}$ have been used for the immobilization of metal NPs. It is also obvious that the inorganic supports/matrices have led to reduce the catalytic performance due to their rigidity and the greater distance between the surface and functional group. In contrast, organic matrix, templates or amphiphilic polymer matrix have always gained significant attention as they are inert, non-toxic, non-volatile, stable, flexible and reusable. ${ }^{11,12}$

Particularly, substitutions of desired functional groups onto the surface of insoluble polymer beads are more necessary for stabilization of metal NPs. ${ }^{13-15}$ Even though many functional modifications have been performed in the preparation of cross-linked polymer-supported bead matrix but these conventional type of insoluble beads are not popular among the catalytic industries due to various practical reasons. Especially, in terms of efficiency, stability, easy recoverability and handling are generally the expected criteria for 
the synthesis of heterogeneous NP catalysts. Alternatively, in order to fulfill these requirements, initially scientists have prepared insoluble polymer-supported beads by the surface initiator atom transfer radical polymerization technique. ${ }^{16,17}$ These are used as potential matrix for immobilization of metal NPs with higher load and thus enabled to obtained efficient heterogeneous NP catalyst. This technique provides controlled chain growth, well-defined block, graft copolymer and avoided the formation of homopolymer. ${ }^{18}$ Hence, the surface initiated-atom transfer radical polymerization (SI-ATRP) technique is considered to be very efficient for grafting of surface functional groups on solid surfaces such as nanomaterial, fibres, films, membranes and so on. Among the known NPs, silver (Ag), gold (Au) and palladium (Pd) are widely studied because of their characteristic optical, spectroscopic and catalytic properties. Pal and coworkers ${ }^{19}$ reported that the immobilization of AuNPs on an anion exchange matrix and it is used for the reduction of 4-nitrophenol. Among these, an interesting report is the solid phase synthesis of AuNPs on polystyrene matrix through immobilization of protected gold NPs. ${ }^{20}$ They have been extensively exploited in the area of photography, photocatalyst, biological labelling and surface enhanced Raman scattering. Further, it is also known that most of dyes discharged from dye industries are more stable and difficult to biodegrade. ${ }^{21}$

Crystal violet (CV) has been used for various purposes; it is extensively used in textile dying and paper colouring. Now the removal of the textile dyes in waste water discharge from various industries such as the textile, paper, plastic, leather, ink, etc. is an active issue all over the world. There are many classes of dyes such as acidic, basic, neutral, azo, direct, reactive, etc. These dyes can be toxic and potentially carcinogenic. ${ }^{22,23}$ Samji et $a l^{24}$ also studied the photocatalytic degradation of $\mathrm{CV}$ using nanoanatase titanium and $\mathrm{Ag}^{+}$-doped titanium. Therefore, removal of colour from waste water is a major environmental problem and threat to the society. Reactive dyes are hardly eliminated under aerobic conditions and are probably decomposed into carcinogenic aromatic amines under anaerobic conditions. ${ }^{25}$ The conventional methods available for treating the dyes containing waste waters are coagulation, flocculation, ${ }^{26}$ oxidation, ozonation ${ }^{27}$ and membrane separation. ${ }^{28}$ However, these technologies do not show significant efficiency or economic advantage. Further, the existing catalyst cannot be continuously packed into column, batch and continuous flow reactors for continuous operation of degradation of dye pollutants. Therefore, in order to eliminate this problem, it is necessary to fabricate new bead-shaped heterogeneous NP catalysts with higher efficiency, stability, economy, easy synthesis and reusability for potential degradation of dyes available in waste water especially at continuous operation mode. Hence, in this study, we report three types of new bead-shaped heterogeneous NP catalysts, viz., PS-PVBC- $g$-PAC-AgNPs, PS-PVBC- $g$-PAC-AuNPs and PS-PVBC- $g$-PAC-PdNPs for the effective degradation of $\mathrm{CV}$ dyes.

\section{Materials and methods}

\subsection{Materials}

Styrene (St) (Aldrich), vinyl benzyl chloride (VBC) (Aldrich), divinylbenzene (DVB) (Lancaster), gelatin (SD fine), boric acid (SRL), polyvinyl alcohol (SRL), sodium hydroxide (SRL), sodium nitrite (SRL), silver nitrate (SRL), auric acid (Sigma Aldrich), tetrachloropalladate (SRL), ethanol (SRL), sodium borohydride (SD fine) and crystal violet (SRL) were used as such without further purification. Azobisisobutyronitrile (AIBN), dioxane, 1,10-phenanthroline and $\mathrm{CuBr}$ were received from Sigma Chemical. All other chemicals were of analytical grade and were purchased from Merck AG (Darmstadt, Germany). Double distilled water was used for preparation of solutions.

\subsection{Synthesis of new polymer-supported poly(vinyl benzyl} chloride) (PS-PVBC) beads grafted with poly-2-acryloxyethyltrimethylammonium chloride (PAC)

The insoluble cross-linked poly(styrene)-co-poly(vinylbenzylchloride) (PS-PVBC) matrix was prepared by a method reported earlier. ${ }^{29}$ The resulted PS-PVBC beads were grafted with 2-acryloxyethyltrimethylammonium chloride (PAC) via the atom transfer radical polymerization method (ATRP). Initially, PS-PVBC beads (5 g) were swelled in ethanol for $24 \mathrm{~h}$ in $100 \mathrm{ml}$ round bottom flask and then the PAC monomer $(20 \mathrm{ml}, 0.15 \mathrm{~mol}), \mathrm{CuBr}(0.432 \mathrm{~g}$, $3.0 \mathrm{mmol}), 1,10$-phenanthroline $(0.936 \mathrm{~g}, 6.0 \mathrm{mmol})$ and dioxane $(10 \mathrm{ml})$ were added to the flask, then polymerization reaction was carried out at $65^{\circ} \mathrm{C}$ for $18 \mathrm{~h}$ under inert atmosphere. The resulted beads were filtered and then transferred into a $100 \mathrm{ml}$ RB flask, to which $10 \%$ (w/v) of EDTA solution was added and stirred continuously for $24 \mathrm{~h}$ to remove the excess copper ions from the beads. The resulted beads were filtered and washed subsequently with water and ethanol and dried under vacuum at $60^{\circ} \mathrm{C}$ for $24 \mathrm{~h}$. The product, viz, poly(styrene)-polyvinyl benzyl chloride was grafted with 2-acryloxyethyltrimethylammonium chloride (PS-PVBC- $g$-PAC) (scheme 1). The PS-PVBC- $g$-PAC beads were characterized by FT-IR and SEM, the observed spectra and images are shown in figures 1 and 2, respectively. The beads were also analysed with EDAX and the observed results are given in table 1.

\subsection{Preparation of three types of bead-shaped heterogeneous NP catalysts}

The immobilization of AgNPs, AuNPs and PdNPs was performed individually onto the surface of PS-VBC- $g$-PAC polymer matrix by using the respective metal precursors, viz., $\mathrm{AgNO}_{3}, \mathrm{HAuCl}_{4}$ and $\mathrm{K}_{2} \mathrm{PdCl}_{4}$ (scheme 1). ${ }^{29,30}$ That is, $500 \mathrm{mg}$ of PS-VBC- $g$-PAC matrix was taken individually in three different single necked $100 \mathrm{ml}$ round-bottomed flask 


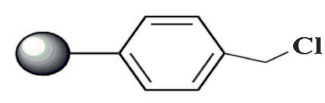

PS-PVBC beads

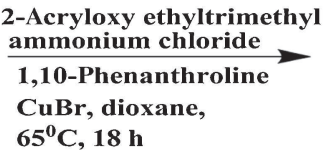

$65^{\circ} \mathrm{C}, 18 \mathrm{~h}$

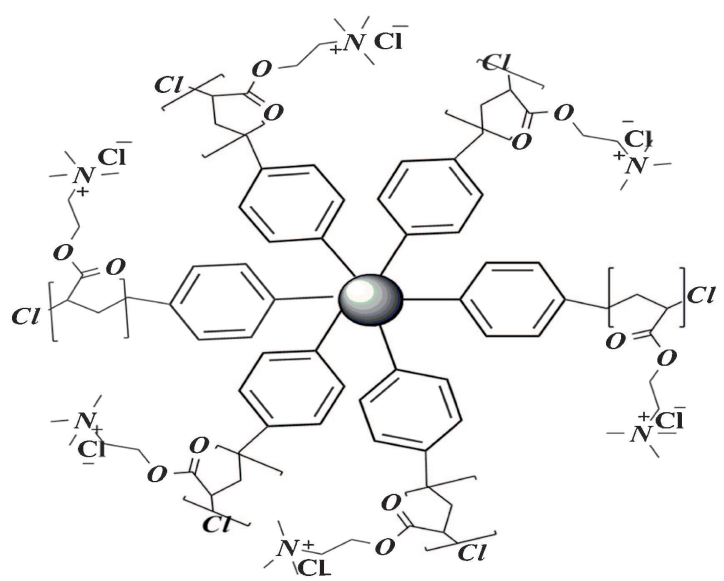

PS-PVBC-g-PAC

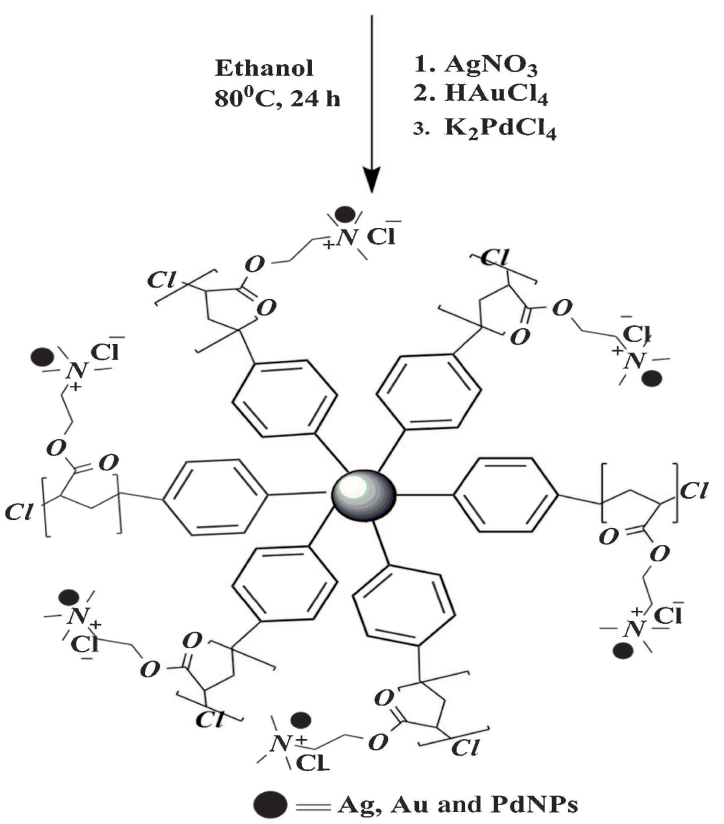

PS-PVBC-g-PAC-AgNPs, AuNPs and PdNPs

Scheme 1. Synthesis of PS-PVBC- $g$-PAC-AgNPs, AuNPs and PdNPs.

and swelled in $50 \mathrm{ml}$ of ethanol overnight. The respective swelled polymer beads were purged with nitrogen for $1 \mathrm{~h}$. After $1 \mathrm{~h}, \mathrm{AgNO}_{3}\left(0.15 \times 10^{-4} \mathrm{~mol}\right)$ was added to one flask, then the solution was refluxed in an oil bath at $80^{\circ} \mathrm{C}$. The reaction mixture has been protected from light to avoid the photochemical reduction of $\mathrm{Ag}^{+}$. After $10 \mathrm{~min}$, the colour of the beads changed to light yellow. Then, 2 equivalents of $\mathrm{NaBH}_{4}$ were added to the solution. Subsequently, after $1 \mathrm{~h}$, the colour was changed from light yellow to dark yellow. After that the reaction mixture was refluxed for $24 \mathrm{~h}$ and then heterogeneous catalysts were filtered through Buchner funnel, washed three times with ethanol and dried in vacuum oven at $60^{\circ} \mathrm{C}$ for $12 \mathrm{~h}$ to yield PS-PVBC- $g$-PAC-AgNPs catalyst.

Similarly, $\mathrm{HAuCl}_{4}\left(0.15 \times 10^{-4} \mathrm{~mol}\right)$ solution was added to the swelled PS-VBC- $g$-PAC beads, and it was observed that the colour of the solution changed from colourless to yellow. Then 2 equivalents of $\mathrm{NaBH}_{4}$ was added to the flask which in turn changed the yellow colour beads into dark purple. The beads were refluxed for $24 \mathrm{~h}$ and then filtered, washed three times with ethanol and dried in vacuum oven at $60^{\circ} \mathrm{C}$ for $12 \mathrm{~h}$ to yield AuNPs immobilized PS-VBC- $g$-PAC heterogeneous NPs catalyst, viz., PS-PVBC- $g$-PAC-AuNPs.

Similarly, heterogeneous palladium NP catalyst was synthesized by adding $\mathrm{K}_{2} \mathrm{PdCl}_{4}\left(0.15 \times 10^{-4} \mathrm{~mol}\right)$ solution to the swelled PS-VBC- $g$-PAC beads. The colour of the solution changed from colourless to yellow, indicating the complexation of $\mathrm{Pd}^{2+}$ onto the poly(2-acryloxyethyltrimethylammonium chloride) (PAC). The resultant PS-PVBC- $g$ PAC-Pd ${ }^{2+}$ complex were treated for reduction reaction by adding 2 equivalents of $\mathrm{NaBH}_{4}$. The yellow coloured beads turned into intense brown and were refluxed for $24 \mathrm{~h}$ and filtered, washed three times with ethanol and dried in vacuum oven at $60^{\circ} \mathrm{C}$ for $12 \mathrm{~h}$ to yield PdNPs immobilized PS-VBC$g$-PAC heterogeneous NPs catalyst, viz., PS-PVBC- $g$-PACPdNPs. 


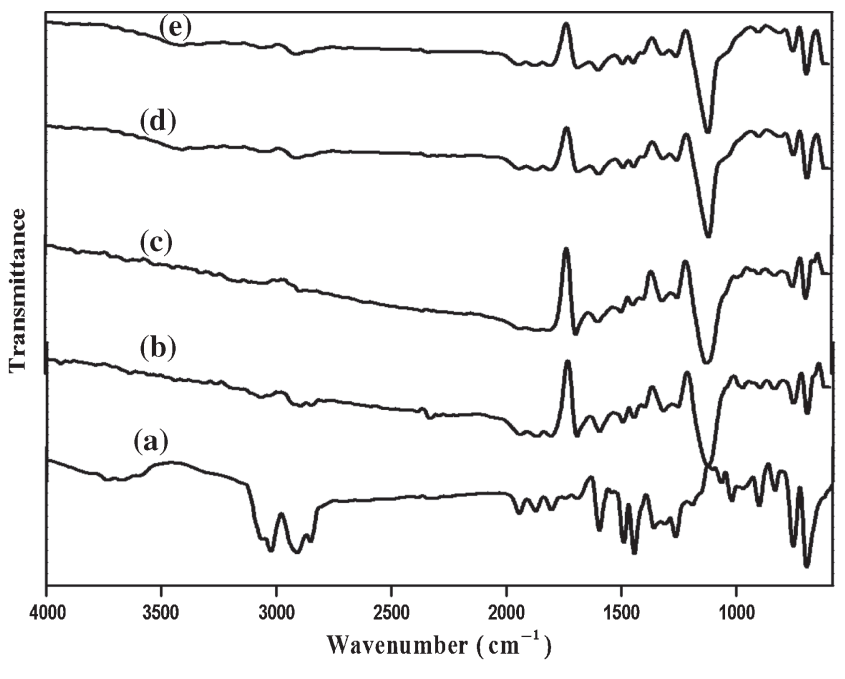

Figure 1. FT-IR spectra of (a) PS-PVBC, (b) PS-PVBC- $g$-PAC, (c) PS-PVBC- $g$-PAC-AgNPs, (d) PS-PVBC- $g$-PAC-AuNPs and (e) PS-PVBC- $g$-PAC-PdNPs.

The newly developed three catalysts, viz., PS-PVBC- $g$ PAC-AgNPs, PS-PVBC- $g$-PAC-AuNPs and PS-PVBC- $g$ PAC-PdNPs were characterized by FT-IR and UV-Vis and the observed spectra are shown in figures 1 and 3, respectively. Similarly, the surface morphology and immobilization of AgNPs, AuNPs and PdNPs onto the respective matrix were studied with SEM, EDAX and HRTEM analyses. The observed SEM and HRTEM images for all the three catalysts are shown in figures 2 and 4, and the observed EDAX results are given in table 1 .

\subsection{Catalysis of PS-PVBC-g-PAC-AgNPs, PS-PVBC-g- $P A C-A u N P s$ and $P S-P V B C-g-P A C-P d N P$ s catalysts in reduction of $C V$}

The comparative catalytic efficiency of PS-PVBC- $g$-PACAgNPs, PS-PVBC- $g$-PAC-AuNPs and PS-PVBC- $g$-PACPdNPs catalysts were examined using reduction of $\mathrm{CV}$ as a model reaction keeping under pseudo-first-order reaction conditions. The individual kinetic experiment was performed by taking three different $25 \mathrm{ml}$ two necked RB flasks to which $10 \mathrm{ml}$ of $\mathrm{CV}\left(1.0 \times 10^{-4} \mathrm{~mol}\right), 10 \mathrm{ml}$ of aqueous $\mathrm{NaBH}_{4}\left(1.0 \times 10^{-2} \mathrm{~mol}\right)$ and $25 \mathrm{mg}$ of the respective catalyst, viz., PS-PVBC- $g$-PAC-AgNPs, PS-PVBC- $g$ PAC-AuNPs and PS-PVBC- $g$-PAC-PdNPs were added to the respective container. The corresponding reaction medium was agitated by stirring at ambient temperature. The progress of the reactions in each container was monitored by withdrawing the respective reaction sample $(3 \mathrm{ml})$ under equal interval of times ( $5 \mathrm{~min}$ ) and was analysed through UVVis spectrophotometer (figure 5). The decreasing trends of absorbance at $586 \mathrm{~nm}$ indicates that the reduction of $-\mathrm{C}=\mathrm{N}$ group in $\mathrm{CV}$ and the rate constant was calculated using the following formula irrespective of the reaction:

$$
k_{\mathrm{obs}}=\ln \left[\left(A_{\infty}-A_{0}\right) /\left(A_{\infty}-A_{\mathrm{t}}\right)\right] / t,
$$

where $A_{0}$ is the initial absorbance, $A_{\mathrm{t}}$ the absorbance at time ' $t$ ', $A_{\infty}$ the absorbance at reaction completion.

\section{Results and discussion}

Three types of new bead-shaped heterogeneous NP catalysts, $v i z$. PS-PVBC- $g$-PAC-AgNPs, PS-PVBC- $g$-PAC-AuNPs and PS-PVBC- $g$-PAC-PdNPs were prepared by adopting the simplified experimental route and thus characterized with FT-IR, UV-Vis, HRTEM, SEM and EDAX. The catalytic performance of these catalysts was examined through the reduction of $\mathrm{CV}$ as a model reaction performed under pseudo-first-order reaction conditions.

\subsection{Characterization of $P S-P V B C-g-P A C-A g N P s$, $P S-P V B C-g-P A C-A u N P s$ and $P S-P V B C-g-P A C-P d N P s$}

The FT-IR spectra obtained from PS-PVBC, PVBC- $g$ PAC, PS-PVBC- $g$-PAC-AgNPs, PS-PVBC- $g$-PAC-AuNPs and PS-PVBC- $g$-PAC-PdNPs are shown in figure 1a-e. From the FT-IR spectrum of the PS-PVBC beads, the $\mathrm{C}-\mathrm{Cl}$ stretching frequency was noticed at $698 \mathrm{~cm}^{-1}$ (figure 1a) which confirms the presence of $\mathrm{CH}_{2}-\mathrm{Cl}$ group onto the surface of polymer beads. ${ }^{32}$ The functionalization of PAC onto PS-PVBC matrix was established from the appearance of $\mathrm{C}=0_{(\mathrm{str})}$ and $\mathrm{C}-\mathrm{N}_{(\mathrm{str})}^{+}$peaks at 1710 and $1121 \mathrm{~cm}^{-1}$ (figure 1b), respectively. Similarly, the formation/immobilization of AgNPs, AuNPs and PdNPs onto the respective matrices was also ascertained from FT-IR results. That is, after individual immobilization of AgNPs, AuNPs and PdNPs, the characteristic peak for $\mathrm{C}-\mathrm{N}$ stretching $\left(1121 \mathrm{~cm}^{-1}\right)$ was shifted to 1126,1113 and $1117 \mathrm{~cm}^{-1}$, respectively, and thus confirmed the formation/immobilization of AgNPs, AuNPs and PdNPs onto the respective matrices.

The reduction/formation of $\mathrm{Ag}, \mathrm{Au}$ and PdNPs immobilized onto the respective PS-PVBC- $g$-PAC-AgNPs, PSPVBC- $g$-PAC-AuNPs and PS-PVBC- $g$-PAC-PdNPs catalysts were inspected by UV-Vis analysis. The recorded spectra were shown in figure $3 \mathrm{a}-\mathrm{c}$. From the figure $3 \mathrm{a}-\mathrm{c}$, it is observed that, clear and sharp peak was noticed at 405, 536 and $240 \mathrm{~nm}$ for $\mathrm{Ag},{ }^{33} \mathrm{Au}^{30,34,35}$ and PdNPs,${ }^{36}$ respectively. The appearance of these surface plasma resonance peaks noticed in the respective filtrate derived from PS-PVBC- $g$ PAC-AgNPs, PS-PVBC- $g$-PAC-AuNPs and PS-PVBC- $g$ PAC-PdNPs strongly confirms the formation/immobilization of AgNPs, AuNPs and PdNPs onto the respective catalyst. Further, the HRTEM images were showed the homogeneous distribution of MNPs with nano in size ranging from 20 to $25 \mathrm{~nm}$ and these observations were strongly evidenced for the immobilization of AgNPs, AuNPs and PdNPs onto the respective PS-PVBC- $g$-PAC matrices.

SEM analysis was performed for PS-PVBC, PS-PVBC$g$-PAC-AgNPs, PS-PVBC- $g$-PAC-AuNPs and PS-PVBC$g$-PAC-PdNPs (figure 2a-h) catalysts with lower (figure 2c, $\mathrm{e}$ and $\mathrm{g}$ ) and higher magnification (figure $2 \mathrm{~d}, \mathrm{f}$ and $\mathrm{h}$ ). In figure 2a, the SEM image of PS-PVBC shows a smooth 

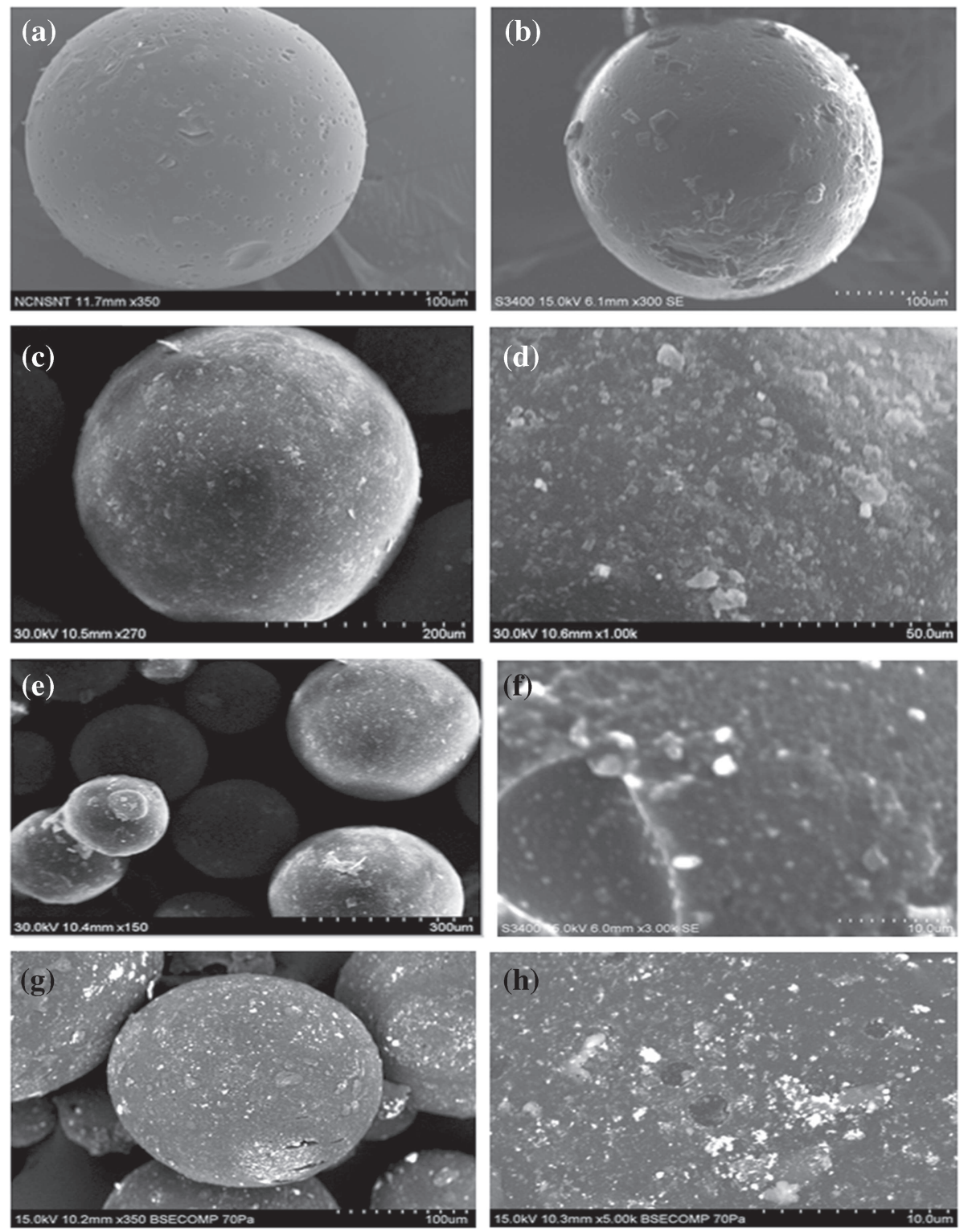

Figure 2. SEM images of (a) PS-PVBC, (b) PS-PVBC-g-PAC, (c) PS-PVBC- $g$-PACAgNPs (low magnification), (d) PS-PVBC-g-PAC-AgNPs (high magnification), (e) PSPVBC- $g$-PAC-AuNPs (low magnification), (f) PS-PVBC- $g$-PAC-AuNPs (high magnification), (g) PS-PVBC- $g$-PAC-PdNPs (low magnification) and (h) PS-PVBC-g-PAC-PdNPs (high magnification).

Table 1. Percentage of elements determined from EDAX analysis using polymer-supported AgNPs, AuNPs and PdNPs.

\begin{tabular}{llllllll}
\hline Name of the compound & $\mathrm{C} \%$ & $\mathrm{~N} \%$ & $\mathrm{Cl} \%$ & $\mathrm{O} \%$ & AgNPs $\%$ & AuNPs $\%$ & PdNPs $\%$ \\
\hline PS-PVBC- $g$-PAC & 65.44 & 21.09 & 6.12 & 7.30 & - & - & - \\
PS-PVBC- $g$-PAC-AgNPs & 76.53 & 14.3 & 5.52 & 3.28 & 0.37 & - & - \\
PS-PVBC- $g$-PAC-AuNPs & 72.46 & 16.3 & 4.94 & 5.86 & - & 0.44 & - \\
PS-PVBC- $g$-PAC-PdNPs & 75.03 & 17.14 & 3.4 & 4.06 & - & - & 0.30 \\
PS-PVBC- $g$-PAC-AuNPs (recycle catalyst) & 73.45 & 16.81 & 5.11 & 4.24 & - & 0.40 & - \\
\hline
\end{tabular}

and homogeneous surface. The grafting of PAC onto the surface of PS-PVBC matrix was observed to be a little heterogeneous in nature PS-PVBC (figure 2b). From, figure $2 \mathrm{c}-\mathrm{h}$, there are numerous numbers of white dots were noticed on the surface of PS-PVBC- $g$-PAC matrix which in turn shows the immobilization/stabilization of AgNPs, 
AuNPs and PdNPs onto the matrices. Particularly, the intensity of white dots is more in AuNPs immobilized catalyst than the rest of the catalysts. This observation indicates that the PS-PVBC- $g$-PAC matrix shows higher affinity for attraction/immobilization of AuNPs than with AgNPs and PdNPs. This is due to the fact that the standard reduction potential for gold $(+1.52)$ is greater than silver $(+0.68)$ and palladium

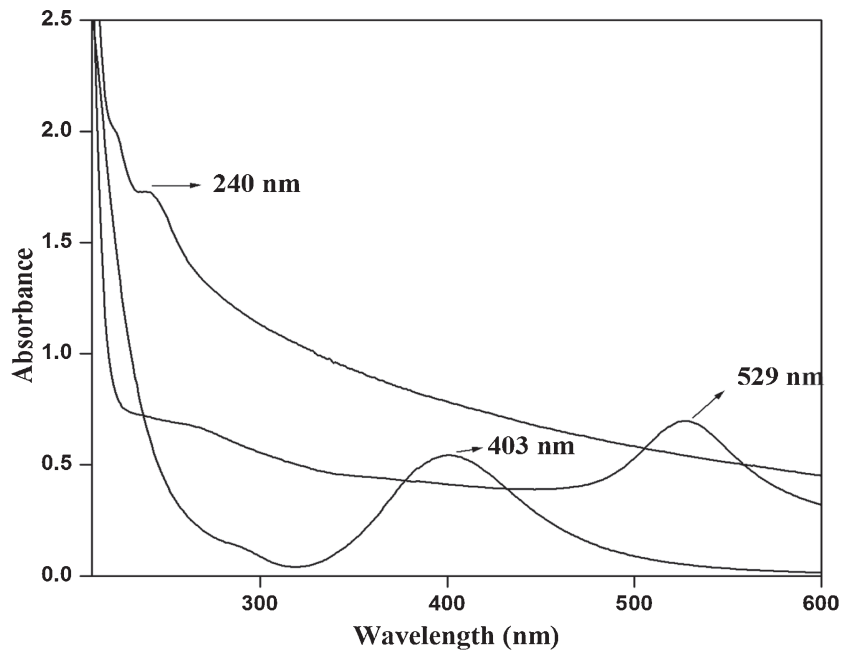

Figure 3. UV-Vis spectra of (a) PS-PVBC- $g$-PAC-AgNPs, (b) PS-PVBC- $g$-PAC-AuNPs and (c) PS-PVBC- $g$-PAC-PdNPs.
$(+0.95)$ and this in turn quickly attract/reduce more number of AuNPs and thus reflecting the presence of more number of white dots in SEM image of PS-PVBC- $g$-PAC-AuNPs.

The immobilization of AgNPs, AuNPs and PdNPs onto the PS-PVBC-g-PAC matrices was studied extensively through EDAX analysis. The characteristic elements such as $\mathrm{C}, \mathrm{N}, \mathrm{O}, \mathrm{Cl}, \mathrm{Ag}, \mathrm{Au}$ and $\mathrm{Pd}$ and their corresponding weight percentage are given in table 1 . The weight percentage of AuNPs was higher than that of the AgNPs and PdNPs, and this in turn confirms the formation/immobilization of more amount AuNPs onto the PS-PVBC- $g$-PAC matrix than with AgNPs and PdNPs. Further, the weight percentage of AuNPs in superior catalyst, viz., PS-PVBC- $g$-PAC-AuNPs obtained from sixth cycle was characterized with EDAX analysis and the weight percentage of the same was found to be 0.40 . Therefore, from the EDAX results, it is observed that there is no loss in weight percentage of AuNPs.

3.2 Comparative study on the catalytic activity of $A g, A u$ and $P d$ immobilized PS-PVBC-g-PAC beads for the reduction of $C V$

The activity of PS-PVBC- $g$-PAC-AgNPs, PS-PVBC- $g$ PAC-AuNPs and PS-PVBC- $g$-PAC-PdNPs catalysts were examined through the reduction of $\mathrm{CV}$ under pseudo-firstorder reaction condition. The reaction was monitored using
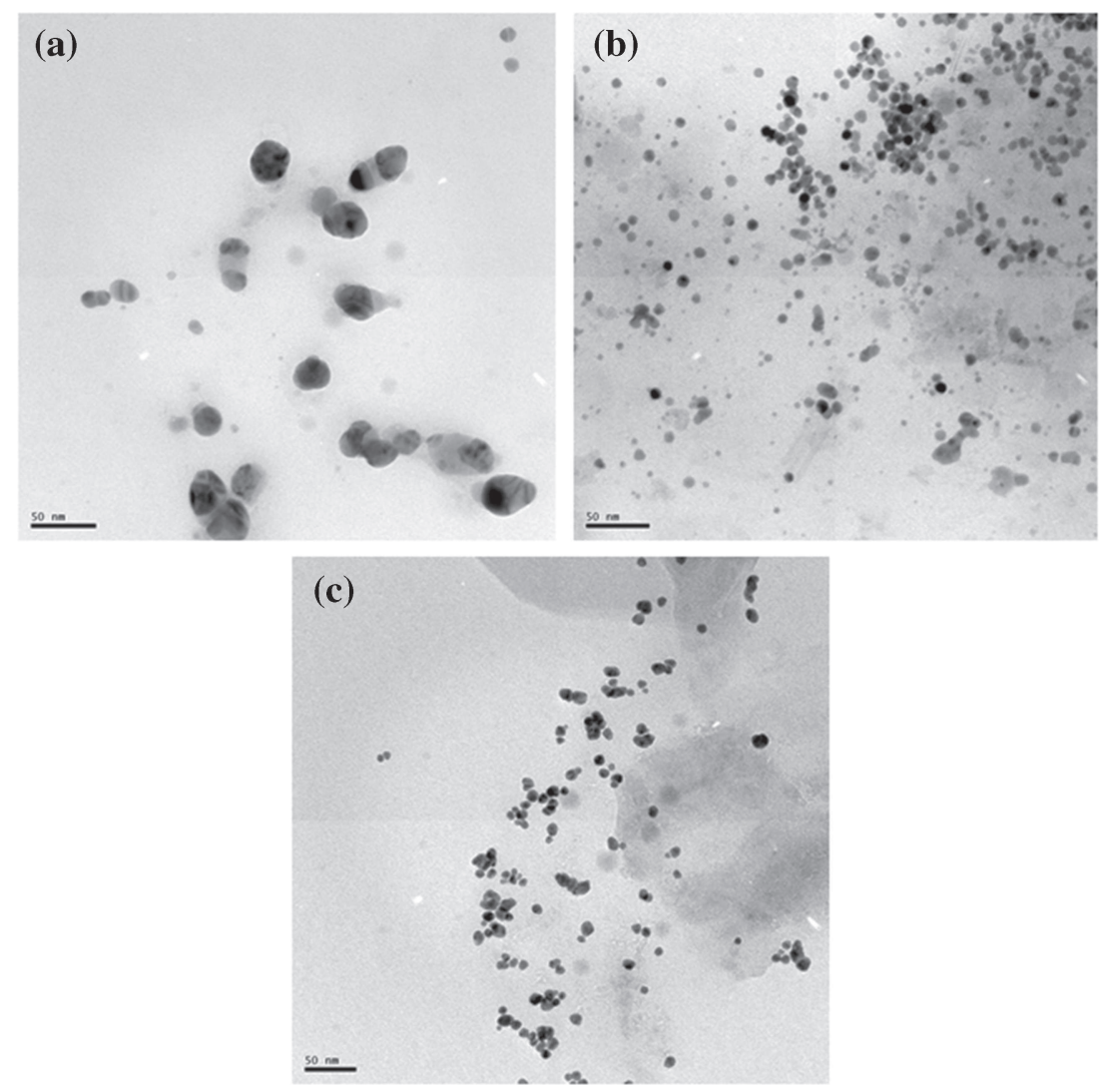

Figure 4. HRTEM images of (a) PS-PVBC- $g$-PAC-AgNPs, (b) PS-PVBC- $g$-PACAuNPs and (c) PS-PVBC- $g$-PAC-PdNPs. 
UV-Vis spectrophotometer by observing the characteristic peak at $586 \mathrm{~nm}$ due to the reduction of $\mathrm{C}=\mathrm{N}^{+}$group in $\mathrm{CV}$ at particular intervals of time $(5 \mathrm{~min})$. From the observed result, the characteristic peak at $586 \mathrm{~nm}$ was found to gradually decrease after adding the respective catalyst, viz., PS-PVBC- $g$-PAC-AgNPs, PS-PVBC- $g$-PAC-AuNPs and PS-PVBC- $g$-PAC-PdNPs and the corresponding spectrum is shown in figure 5. The pseudo-first-order rate constants for the reaction catalysed by PS-PVBC- $g$-PAC-AgNPs, PS-PVBC- $g$-PAC-AuNPs and PS-PVBC- $g$-PAC-PdNPs were determined to be $16.74 \times 10^{4}, 25.46 \times 10^{4}$ and 8.45 $\times 10^{4} \mathrm{~s}^{-1}$, respectively. The observed rate constants shows that PS-PVBC- $g$-PAC-AuNPs catalyst shows better activity than PS-PVBC- $g$-PAC-AgNPs and PS-PVBC- $g$-PACPdNPs catalysts. This is obviously due to the availability

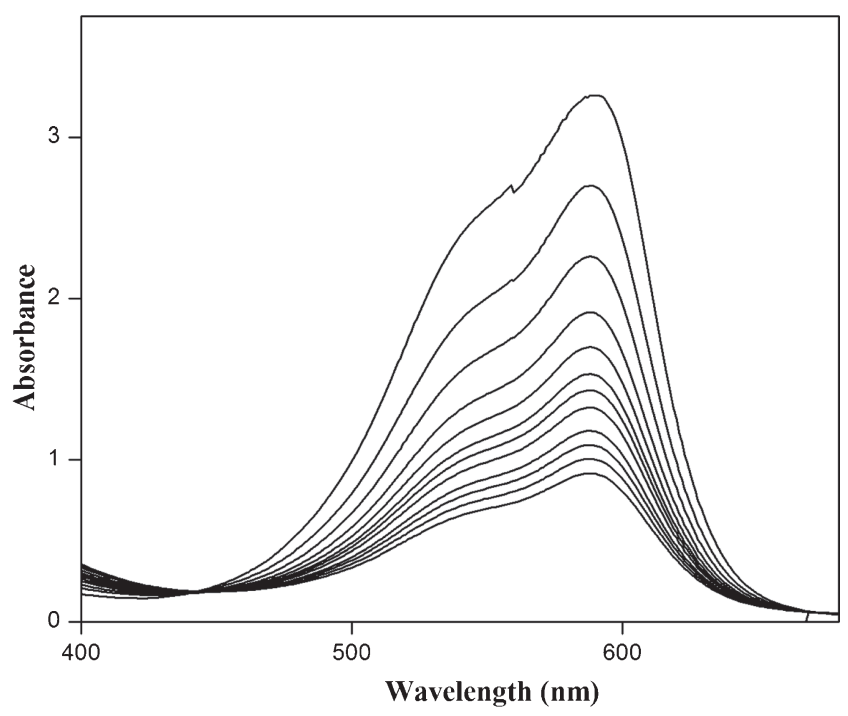

Figure 5. UV-Vis spectra for the reduction of $\mathrm{CV}$ using PS-PVBC- $g$-PAC-AuNPs.

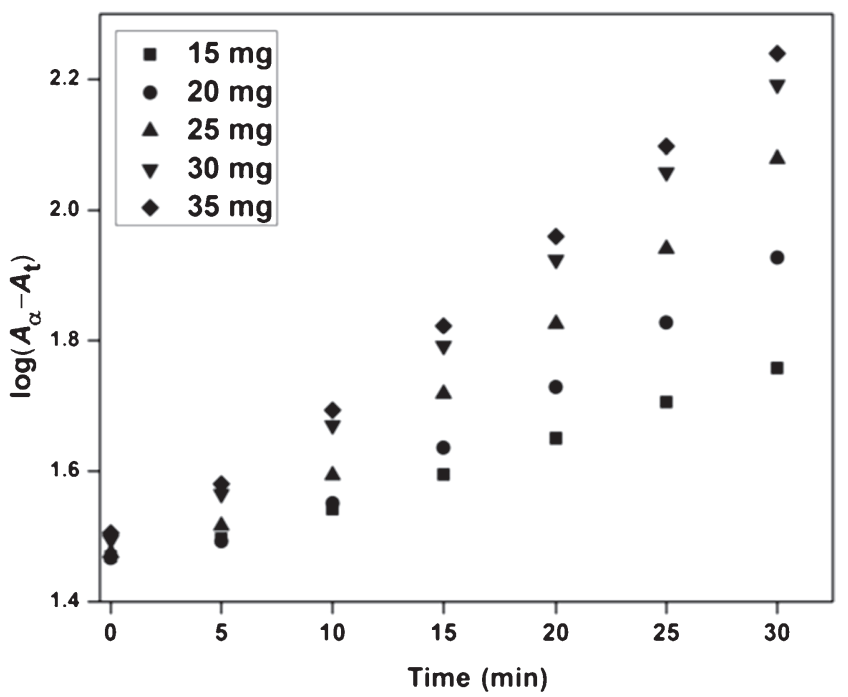

Figure 6. Effect of [catalyst]. of more number of AuNPs stabilization onto the surface of PS-PVB- $g$-PAC. Further, the more number of AuNPs stabilization/immobilization is possible because of grafting of quaternary onium group achieved through the SI-ATRP technique. Thorough kinetic studies was performed with superior catalyst, viz., PS-PVBC-g-PAC-AuNPs by varying the parameters such as [substrate], [catalyst] and $\left[\mathrm{NaBH}_{4}\right]$ under pseudo-first-order condition. It was observed that, the rate constant was proportional to the amount of catalyst, viz. , PSPVBC- $g$-PAC-AuNPs. This may be attributed to the increase in the number and availability of catalyst NPs with higher catalyst loading. On increasing the $\left[\mathrm{NaBH}_{4}\right]$, the rate constant was also found to increase which should due to more adsorption of $\mathrm{NaBH}_{4}$ onto the NP surfaces, which in turn increases the relay of more electrons from $\mathrm{BH}_{4}^{-}$(donor) to the $\mathrm{CV}$ (receptor). At higher [CV], the intensities of penetration of light inside the solutions were found to be decreased

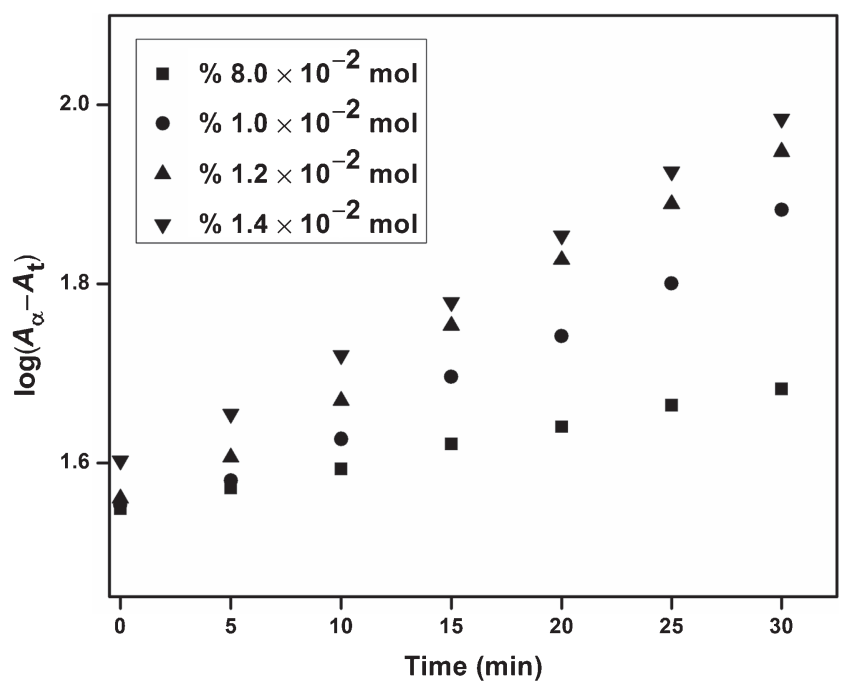

Figure 7. Effect of $\left[\mathrm{NaBH}_{4}\right]$.

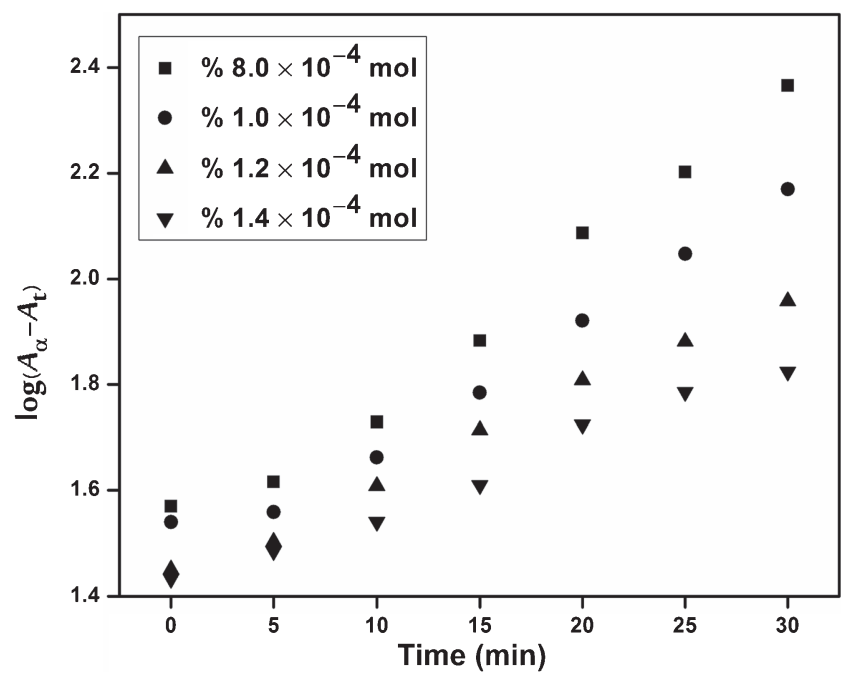

Figure 8. Effect of [substrate]. 
Table 2. Comparative catalytic study of PS-PVBC- $g$-PAC stabilized MNPs catalyst reduction of CV and recycling efficiency PS-PVBC$g$-PAC-AuNPs.

\begin{tabular}{|c|c|c|c|c|c|c|c|}
\hline \multirow[b]{2}{*}{ S. no. } & \multirow[b]{2}{*}{ Name of the catalyst } & \multirow{2}{*}{$\begin{array}{c}k_{\mathrm{obs}} \times 10^{4} \mathrm{~s}^{-1} \\
\text { Reduction of } \mathrm{CV}\end{array}$} & \multicolumn{5}{|c|}{ Recycling efficiency of PS-PVBC- $g$-PAC-AuNPs } \\
\hline & & & Cycle-2 & Cycle-3 & Cycle-4 & Cycle-5 & Cycle-6 \\
\hline 1 & PS-PVBC- $g$-PAC-AgNPs & 16.74 & - & - & - & - & - \\
\hline 2 & PS-PVBC- $g$-PAC-AuNPs & 25.46 & 25.39 & 25.35 & 25.41 & 25.27 & 25.19 \\
\hline 3 & PS-PVBC- $g$-PAC-PdNPs & 08.45 & - & - & - & - & - \\
\hline
\end{tabular}

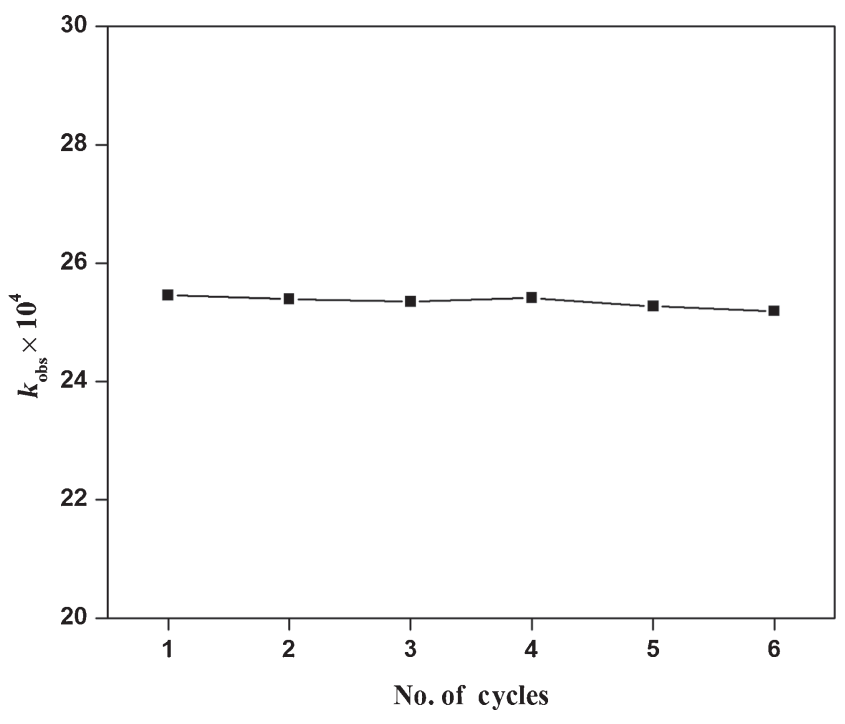

Figure 9. Recycling efficiency of PS-PVBC- $g$-PAC-AuNPs catalyst.

which in turn decreases the rate constant. The pseudo-firstorder rate constants were evaluated and plotted against the [catalyst], $\left[\mathrm{NaBH}_{4}\right]$ and [substrate] and the corresponding plots are shown in figures 6-8.

\subsection{Recycling efficiency of PS-PVBC-g-PAC-AuNPs}

In order to examine the stability/reusability of the catalyst, the PS-PVBC- $g$-PAC-AuNPs catalyst was filtered off and washed thoroughly with distilled water, dried at room temperature and employed upto 6 cycles for reduction of $\mathrm{CV}$ under same reaction condition. The observed rate constants are given in table 2. It is seen that the observed rate constant remains constant and thus confirmed the stability of the catalyst (figure 9).

\section{Conclusion}

Three types of new beads shaped heterogeneous nanoparticle catalysts, viz., PS-PVBC- $g$-PAC-AgNPs, PS-PVBC$g$-PAC-AuNPs and PS-PVBC- $g$-PAC-PdNPs were synthesized by a simplified procedure. All the three catalysts were characterized by spectroscopy and microscopy techniques.
The appearance of new peak at $1121 \mathrm{~cm}^{-1}$ in FT-IR was confirmed the grafting of PAC in PS-PVBC matrix and SPR peaks noticed at 405,536 and $240 \mathrm{~nm}$ in UV-Vis spectra were confirmed the formation/immobilization of AgNPs, AuNPs and PdNPs onto the surface of PS-PVBC- $g$-PAC matrix, respectively. The catalytic activity was inspected using the reduction of $\mathrm{CV}$ as a model reaction keeping under pseudo-first-order reaction conditions. From the calculated $k_{\mathrm{obs}}\left(k_{\mathrm{obs}}=25.46 \mathrm{~s}^{-1}\right)$ values, it is observed that the PSPVBC- $g$-PAC-AuNPs catalyst was more active than PSPVBC- $g$-PAC-AgNPs $\left(k_{\text {obs }}=16.74 \mathrm{~s}^{-1}\right)$ and PS-PVBC$g$-PAC-PdNPs catalyst $\left(k_{\mathrm{obs}}=8.45 \mathrm{~s}^{-1}\right)$. Further, the thorough kinetic study was conducted using the superior catalyst, viz., PS-PVBC-g-PAC-AuNPs by varying the parameters such as [catalyst], [substrate] and $\left[\mathrm{NaBH}_{4}\right]$ and it was observed that the rate constant is dependent on each parameter. The filtered PS-PVBC- $g$-PAC-AuNPs catalyst was reused again the same reaction and it was noticed that the activity was maintained upto sixth cycle and thus proved the efficiency/stability of the catalyst.

\section{Acknowledgements}

We thank the University of Madras, for financial support under the scheme URF and also to NCNSNT, University of Madras, for providing instrumentation facilities.

\section{References}

1. Storhoff J J, Elghanian R, Mucic R C, Mirkin C A and Letsinger R L 1998 J. Am. Chem. Soc. 1201959

2. Zhao M, Sun L and Crooks R M 1998 J. Am. Chem. Soc. 120 4877

3. Liu Z, Lee J Y, Chen W, Han M and Gan L M 2004 Langmuir 20181

4. Ertl G, Kneozinger H and Weitkamp J 1997 Handbook of heterogeneous catalysis (Weinheim: $\mathrm{VCH}$ )

5. Yoo J W, Hathcock D J and El-Sayed M A 2003 J. Catal. 2141

6. Mallick K and Scurrell M S 2003 Appl. Catal. A: Gen. 253527

7. Suzuki K, Yumura T, Mizuguchi M, Tanaka Y, Chen C W and Akashi M J 2000 J. Polym. Sci. A 772678

8. Boudjahem A G, Monteverdi S, Mercy M and Bettahar M M 2004 J. Catal. 221325

9. Chen C W, Chen M Q, Serizawa T and Akashi M 1998 Chem. Commun. 7831 
10. Peng L, Yushan L and Zhixing S 2006 Ind. Eng. Chem. Res. 45 2255

11. Burguete M I, Fraile J M, Garcia-Verdugo E, Luis S V, Martinez-M V and Mayoral J A 2005 Ind. Eng. Chem. Res. 44 8580

12. Amy S H King and Lance J Twyman 2002 J. Chem. Soc., Perkin Trans. 12209

13. Liu W, Yang X and Huang W J 2006 Colloids Interface Sci. 304160

14. Ou J L, Chang C P, Sung Y, Ou K L, Tseng C C, Ling H W and Ger M D 2007 Colloids Surf. A 30536

15. Wu H, Liu Z, Wang X, Zhao B, Zhang J and Li C J $2006 \mathrm{~J}$. Colloid Interface Sci. 302142

16. Yavuz E, Bayramoglu G, Senkal B F and Yakup Arica M 2009 J. Appl. Polym. Sci. 1132661

17. Shipp D A, Wang J L and Matjaszewski K 1998 Macromolecules $\mathbf{3 1} 8005$

18. Grubbs R B, Hawker C J, Dao J and Frechet J M 1997 Angew. Chem., Int. Ed. Engl. 36270

19. Praharaj S, Nath S, Ghosh S K, Kundu S and Pal T 2004 Langmuir 209889

20. Youk J H 2003 Polymer 445053

21. Seshadri S, Bishop PL and Agha A M 1994 Waste Manage 14127

22. Chun H, Yuchao T, Jimmy C Y and Wonga P K 2003 Appl. Catal. B-Environ. 40131
23. Hu C, Yu J C, Hao Z and Wong P K 2003 Appl. Catal. BEnviron. 4635

24. Samji S, Akash R, Mohan J M and Modak J 2012 Thermodynam. Catal. 35

25. Peterson D 1990 in: Colorants and auxiliaries: organic chemistry and application properties. J Shore (ed) (Manchester: BTTG-Shirley) vol. 1, p 32

26. Panswad T and Wongchaisuwan S 1986 Water Sci. Technol. 18139

27. Malik P K and Saha S K 2003 Sep. Purif. Technol. 31241

28. Abid M F, Zablouk M A and Abid-Alameer A M 2012 Iranian J. Environ. Health Sci. Eng. 917

29. Murugan E and Jebaranjitham J N 2012 J. Mol. Catal. A: Chem. 365128

30. Murugan E and Rangasamy R 2010 J. Polym. Sci., Part A: Polym. Chem. 482525

31. Murugan E and Gopinath P 2007 Appl. Catal. A 31972

32. Murugan E and Vimala G 2013 J. Colloid Interface Sci. 396101

33. Murugan E and Jebaranjitham J N 2011 J. Biomed. Nanotechnol. 7158

34. Murugan E, Rangasamy R and Nimita Jebaranjitham J 2012 Adv. Sci. Lett. 6250

35. Murugan E, Rangasamy R and Pakrudheen I 2012 Sci. Adv. Mater. 41103

36. Yong-M L, Hai-Z Z, Wei-G L, Bo H and Shu-H Y 2013 J. Mater. Chem. A 13783 